\title{
Circulating metabolites from the choline pathway and acute coronary syndromes in a Chinese case-control study
}

Yuxiang Dai ${ }^{1}$, Qianqian Tian 1,2,3, Jing Si ${ }^{4}$, Zhonghan Sun², Shalaimaiti Shali', Lili Xu', Daoyuan Ren', Shufu Chang ${ }^{1}$, Xin Dong ${ }^{1,5}$, Hongxia Zhao ${ }^{1,5}$, Zhendong $\mathrm{Mei}^{2}$, Yan Zheng ${ }^{1,2,4^{*}}$ and Junbo Ge ${ }^{\text {* }^{*}}$

\begin{abstract}
Background: Accumulating evidence shows that circulating levels of trimethylamine $\mathrm{N}$-oxide, which is generated from the metabolism of dietary choline, may predict cardiovascular disease among Caucasians. Acute coronary syndrome (ACS), one common presentation of cardiovascular disease, is a spectrum of signs and symptoms due to acute decreased blood flow in the coronary arteries. The relationship between the metabolites from choline pathway and ACS remains unclear. We aimed to assess the associations of circulating metabolites from the choline pathway with ACS among a Chinese population, who consume a different dietary pattern than their Western counterparts.
\end{abstract}

Methods: We recruited 501 participants who were admitted to the Department of Cardiology, Zhongshan Hospital,Shanghai China between March 2017 and June 2018, including 254 ACS cases and 247 controls. Liquid chromatography-tandem mass spectrometry was used to measure circulating concentrations of metabolites in the choline pathway, including betaine, choline, trimethylamine, and trimethylamine $\mathrm{N}$-oxide. A composite metabolite score using a weighted sum of these four metabolites, and the betaine/choline ratio were calculated. Multivariable logistic regressions were applied to estimate the association of metabolites with ACS, with adjustment of age, sex, body mass index, smoking index, history of diseases, and kidney function.

Results: After adjusting for traditional risk factors, per 1-standard deviation (SD) increment in choline was positively associated with the odds of ACS [odds ratio (OR), 95\% confidence interval (CI), 1.77(1.44-2.18)], and the other metabolites were not associated with ACS at a statistical significance level. Compared with participants in the lowest quartile of the metabolite score, those in the highest quartile had higher odds of ACS [OR $(95 \% \mathrm{Cl})$, 3.18(1.85-5.54), $p<0.001$ for trend]. Per 1-SD increment in metabolite score was positively associated with higher odds of ACS [OR $(95 \% \mathrm{Cl}), 1.80(1.37-2.40)]$, and per 1-SD increment in the betaine/choline ratio was inversely associated with the odds of ACS [OR (95\% Cl), 0.49 (0.39-0.60)].

(Continued on next page)

\footnotetext{
* Correspondence: yan_zheng@fudan.edu.cn; jbge@zs-hospital.sh.cn

'Department of Cardiology, Shanghai Institute of Cardiovascular Disease,

ZhongShan Hospital, Fudan University, 1609 Xietu Road, Shanghai 200032,

China

Full list of author information is available at the end of the article
}

(c) The Author(s). 2020 Open Access This article is licensed under a Creative Commons Attribution 4.0 International License, which permits use, sharing, adaptation, distribution and reproduction in any medium or format, as long as you give appropriate credit to the original author(s) and the source, provide a link to the Creative Commons licence, and indicate if changes were made. The images or other third party material in this article are included in the article's Creative Commons licence, unless indicated otherwise in a credit line to the material. If material is not included in the article's Creative Commons licence and your intended use is not permitted by statutory regulation or exceeds the permitted use, you will need to obtain permission directly from the copyright holder. To view a copy of this licence, visit http://creativecommons.org/licenses/by/4.0/ The Creative Commons Public Domain Dedication waiver (http://creativecommons.org/publicdomain/zero/1.0/) applies to the data made available in this article, unless otherwise stated in a credit line to the data. 
(Continued from previous page)

Conclusions: Among our Chinese participants, trimethylamine N-oxide was not associated with ACS, while a composite metabolite score of metabolites from the choline pathway was associated with increased odds of ACS. The choline pathway metabolites may be related to the pathophysiology of ACS among Chinese.

Keywords: Choline, Cardiovascular risk, Metabolite score, Trimethylamine N-oxide, Intestinal flora

\section{Introduction}

Acute coronary syndrome (ACS), a common subcategory of cardiovascular disease (CVD), has led to increased mortality globally [1]. ACS is a set of signs and symptoms due to acutely decreased blood flow in the coronary arteries, and the exact mechanism underlying its pathogenesis remains to be fully elucidated. Emerging metabolomics studies have provided novel pathways of CVD development, for example, intestinal microbiota related metabolites may play important roles in the progression of atherosclerosis $[2,3]$. Remarkably, elevated levels of trimethylamine $\mathrm{N}$-oxide (TMAO), a metabolite generated from the metabolism of dietary choline, carnitine and phosphatidylcholine (mostly originated from red meat, eggs, and fish) by gut microbiome [4], and its precursors were associated with increased risk of major adverse cardiovascular events in Western and European cohorts [5-7]. Thus far, little evidence exists regarding whether these metabolites are associated with ACS.

Different studies presented relatively heterogeneous results regarding metabolites from choline pathway and CVD $[8,9]$, and one possible reason could be different population structure and distinct dietary patterns [8]. Evidence from Chinese populations is in lack compared to the Caucasians. Moreover, most studies failed to assess the relationship between the combinded effect of circulating metabolites in the choline pathway and CVD. Hence, in this study, we sought to quantify four circulating metabolites from choline pathway including betaine, choline, trimethylamine (TMA) and TMAO, and to estimate the associations between choline pathway metabolites and ACS among Chinese participants.

\section{Methods}

\section{Study population and biomedical measurements}

A total of 501 participants were recruited from the Department of Cardiology, Zhongshan Hospital Shanghai China, between March 2017 and June 2018, including 254 cases and 247 controls. Cases were clinically diagnosed as ACS with documented $\geq 50 \%$ stenosis of at least one epicardial coronary artery during the coronary angiography $[10,11]$, and controls were non-ACS participants who underwent coronary angiography with a normal coronary artery. Each participant provided written informed consent. The study was approved by the Ethics Committee of the Zhongshan Hospital Fudan University.

Data on demographics (age and gender), anthropometrics (height and weight), lifestyle (smoking), history of disease (i.e., hypertension, diabetes mellitus and hyperlipidemia), kidney function [estimated glomerular filtration rate (eGFR)] were collected from the electronic medical record systems in Department of Cardiology. The severity of coronary atherosclerosis was assessed using the Gensini score, a widely used scoring system to quantify coronary atherosclerosis burden, in which a zero score indicates absence of atherosclerotic disease, and a higher score accounts for a severer proximal lesion by combining the degree of luminal narrowing as well as the location of narrowing $[12,13]$.

Fasting blood samples were collected using tubes containing EDTA via radial access before heparinization and then immediately stored at $-80^{\circ} \mathrm{C}$ until analysis. Plasma proteins were precipitated with 3 volumes of methanol containing a mixed internal standard of $500 \mathrm{ng} / \mathrm{ml}$. After vortex and centrifugation, supernatants were analyzed with an Agilent 1290 Infinity UHPLC instrument (Agilent, USA) on an XBridge BEH Hilic Column $(2.5 \mu \mathrm{m}$, $2.1 \times 100 \mathrm{~mm}$, Waters, Milford, MA) at a flow rate of $0.35 \mathrm{ml} / \mathrm{min}$. LC gradient was starting from $2 \% 10 \mathrm{mM}$ ammonium formate (A) and $98 \%$ acetonitrile of (B, $\mathrm{PH} 3.5)$ over $1 \mathrm{~min}$, then increased to $10 \% \mathrm{~A}$ at $6 \mathrm{~min}$, holding $1 \mathrm{~min}$; then to $15 \% \mathrm{~A}$ at $10 \mathrm{~min}, 30 \% \mathrm{~A}$ at 12 min and $40 \% \mathrm{~A}$ at $13 \mathrm{~min}$. The supernatants of $20 \mathrm{sam}$ ples were randomly taken out and mixed as SampleQuality Control samples to calculate intra-day relative standard deviations and inter-day relative standard deviations, which were all $<15 \%$ in all the samples for the measured metabolites.

An Agilent 6470 Triple Quadruple (Agilent, USA) equipped with ESI source was used for quantification of TMA, TMAO, choline, and betaine. All the compounds were monitored in positive MRM mode using characteristic precursor-product ion transitions: $\mathrm{m} / \mathrm{z}$ 60.1-44.2, $\mathrm{m} / \mathrm{z}$ 76.1-58.1, m/z 104.1-60.1, m/z 118.1-58.1, m/z 162.1-102.9, m/z 114.0-44.1, respectively. The internal standards TMA-d9, TMAO- $\mathrm{d} 9$, and Choline-d9 were added to blood samples, and monitored in MRM mode at $\mathrm{m} / \mathrm{z} 69.1-49.2, \mathrm{~m} / \mathrm{z} 84.9-68.4$, and $\mathrm{m} / \mathrm{z} 113.1-69.5$, respectively. Series concentrations of TMA, TMAO, choline, and betaine standards and a fixed amount of 
internal standards were spiked into the water to prepare the curves for quantification of blood analytes. L-QC, $\mathrm{M}-\mathrm{QC}$ and $\mathrm{H}-\mathrm{QC}$ were inserted into the sequence to evaluate the accuracy of the method.

The standards for TMA, TMAO, choline and betaine were purchased from Sigma-Aldrich (Shanghai, China). The internal standards for TMA-d9 and TMAO-d9 were obtained from Cambridge Isotope Laboratories, Inc. (MA, USA).

\section{Statistical analyses}

A rank-based inverse normal transformation was applied to approximate the normal distribution of metabolites concentrations [14]. Characteristics were presented as mean (SD) for continuous variables and number (frequencies) for categorical variables. Characteristics in cases and controls were compared using the $t$-test for continuous variables and $X^{2}$-test for categorical variables. Cases were stratified into four groups (quartiles) according to their Gensini scores. We calculated a metabolite score as the weighted sum of levels of four metabolites from the choline pathway: betaine, choline, TMA and TMAO, and modeled the score as the main exposure variable in logistic regressions to estimate the composite association of circulating metabolites in choline pathway with ACS [15]. The weight for each metabolite was the regression coefficient for one SD increment in the blood concentration estimated from the adjusted multivariable logistic regression model. A ratio of betaine to choline was also calculated and modeled as an exposure variable in the regression models. This ratio can be considered as a better predictor of metabolic stress as it combines the predictive power of betaine and choline to metabolic stress together, and it was able to capture the composite associations of betaine and choline with metabolic disturbances [16].

Multivariable (adjusted) logistic regression models were used to evaluate the odds ratios (ORs) and corresponding 95\% confidence intervals (CIs) to estimate the association of circulating metabolites and the metabolite score with the odds of ACS. Circulating metabolites were analyzed as both quartiles (using cut-points defined among controls) and continuous variables (per 1-SD increment). To test the linear trend across quartiles, the median of each quartile was assigned and analyzed as a continuous variable. Logistic regression models were adjusted for age, sex, smoking index (pack-years) and body mass index (BMI) in model 1, model 2 was additionally adjusted for history of the disease (ie., hypertension, diabetes mellitus and hyperlipidemia), and model 3 was further adjusted for kidney function. We used participants assigned to the first quartile of the levels of each metabolite, the metabolite score and betaine to choline ratio, as the reference group in each model. The correlations between circulating metabolites, metabolite score and betaine-to-choline ratio were tested by Spearman correlation. In ACS cases, the relations between circulating metabolites and the coronary atherosclerosis burden measured by Gensini score were also measured.

All statistical analyses and data visualizations were performed using R 3.6.2 (https://www.r-project.org/) and a two-sided $p$ value $<0.05$ was considered statistically significant.

\section{Results}

\section{Characteristics of participants in ACS cases and controls}

The characteristics of the study subjects by ACS status are presented in Table 1 . This study included 430 (85.8\%) men and 71 women, with a mean (SD) age of 62.92 (10.84) years. Compared with the controls, ACS cases were more likely to be current smokers (43.3\%) and diabetes patients $(28.0 \%) \quad(p<0.001)$, and the cases had a significantly higher BMI as expected. There were no significant differences between cases and controls concerning age, the proportions of gender, hypertension and hyperlipidemia as well as the eGFR levels (all $p>0.05$ ).

\section{Differences in circulating metabolites in the choline pathway between the ACS cases and controls}

In this study, ACS cases had significantly higher levels of betaine (mean $\pm \mathrm{SD}, 41.74 \pm 17.87 \mu \mathrm{mol} / \mathrm{L}$ in cases vs. $38.36 \pm 13.55 \mu \mathrm{mol} / \mathrm{L}$ in controls), choline $(57.04 \pm$ $34.70 \mu \mathrm{mol} / \mathrm{L}$ in cases vs. $38.55 \pm 28.99 \mu \mathrm{mol} / \mathrm{L}$ in controls) and metabolite score $(40.21 \pm 22.39$ in cases vs. $28.78 \pm 18.56$ in controls) than controls $(p<0.05$, Table $1)$. There were no significant differences in the levels of TMA and TMAO between cases and controls. A heatmap of Spearman correlation coefficients of the circulating metabolites analyzed in participants was shown in Fig. 1. Negative correlations were observed for choline and betaine-to-choline ratio, and metabolite score and betaine-to-choline ratio $(p<0.001)$.

\section{Circulating concentration of choline pathway metabolites with ACS}

The associations of circulating concentrations of choline pathway metabolites and metabolite score with the odds of ACS were shown in Table 2. Compared with participants in the bottom quartile of choline, participants in the highest quartile of choline had significantly higher odds of ACS after adjusting for traditional risk factors [OR (95\% CI), 3.72 (2.21-6.34), $p<0.001$ for trend]. Per 1-SD increment concentrations of choline were associated with higher odds of ACS [OR (95\% CI), 1.77(1.44-2.18)]. In multivariable-adjusted models, the choline metabolite score was associated with 3.18 fold higher odds of ACS across extreme quartiles [OR (95 
Table 1 Characteristics of participants in cases of acute coronary syndrome and controls

\begin{tabular}{|c|c|c|c|c|}
\hline Characteristics & Total $(n=501)$ & Cases $(n=254)$ & Controls $(n=247)$ & $p$ value \\
\hline Age, mean (SD), year & $62.92 \pm 10.84$ & $63.07 \pm 11.02$ & $62.77 \pm 10.69$ & 0.54 \\
\hline Sex male, No. (\%) & $430(85.8 \%)$ & $217(85.4 \%)$ & $213(86.2 \%)$ & 0.90 \\
\hline Current smokers, No. (\%) & $163(32.5 \%)$ & $110(43.3 \%)$ & $53(21.5 \%)$ & $<0.001$ \\
\hline Hypertension, No. (\%) & $296(59.1 \%)$ & $149(58.7 \%)$ & $147(59.5 \%)$ & 0.92 \\
\hline Diabetes mellitus, No. (\%) & $104(20.8 \%)$ & $71(28.0 \%)$ & $33(13.4 \%)$ & $<0.001$ \\
\hline Hyperlipidaemia, No. (\%) & $13(2.6 \%)$ & $5(2.0 \%)$ & $8(3.2 \%)$ & 0.54 \\
\hline BMl, mean (SD), kg/m² & $25.06 \pm 3.33$ & $24.73 \pm 3.53$ & $25.41 \pm 3.08$ & 0.02 \\
\hline Smoking Index, mean (SD), pack-years & $15.13 \pm 25.21$ & $17.96 \pm 26.37$ & $12.22 \pm 23.73$ & $<0.001$ \\
\hline eGFR, mean (SD), $\mathrm{ml} / \mathrm{min} / 1.73 \mathrm{~m}^{2}$ & $82.36 \pm 19.90$ & $80.76 \pm 20.79$ & $84.01 \pm 18.88$ & 0.07 \\
\hline Gensini Score, mean (SD) & $39.88 \pm 52.01$ & $76.73 \pm 50.83$ & $1.99 \pm 2.90$ & $<0.001$ \\
\hline Betaine, mean (SD), $\mu \mathrm{mol} / \mathrm{L}$ & $40.07 \pm 15.94$ & $41.74 \pm 17.87$ & $38.36 \pm 13.55$ & 0.02 \\
\hline Choline, mean (SD), $\mu \mathrm{mol} / \mathrm{L}$ & $47.93 \pm 33.26$ & $57.04 \pm 34.70$ & $38.55 \pm 28.99$ & $<0.001$ \\
\hline TMA, mean (SD), $\mu \mathrm{mol} / \mathrm{L}$ & $4.10 \pm 2.99$ & $4.27 \pm 3.30$ & $3.93 \pm 2.63$ & 0.20 \\
\hline TMAO, mean (SD), $\mu \mathrm{mol} / \mathrm{L}$ & $1.92 \pm 2.08$ & $1.84 \pm 2.21$ & $1.99 \pm 1.95$ & 0.42 \\
\hline Choline metabolite score, mean (SD) & $34.58 \pm 21.33$ & $40.21 \pm 22.39$ & $28.78 \pm 18.56$ & $<0.001$ \\
\hline Ratio of betaine/choline, mean (SD), $\mu \mathrm{mol} / \mathrm{L}$ & $1.03 \pm 0.44$ & $0.89 \pm 0.43$ & $1.18 \pm 0.40$ & $<0.05$ \\
\hline
\end{tabular}

$B M I$ body mass index, eGFR estimated glomerular filtration rate, TMA trimethylamine, TMAO trimethylamine $\mathrm{N}$-oxide

Choline metabolite score was applied by a weighted sum of concentrations of four metabolites in the choline pathway (betaine, choline, TMA and TMAO)

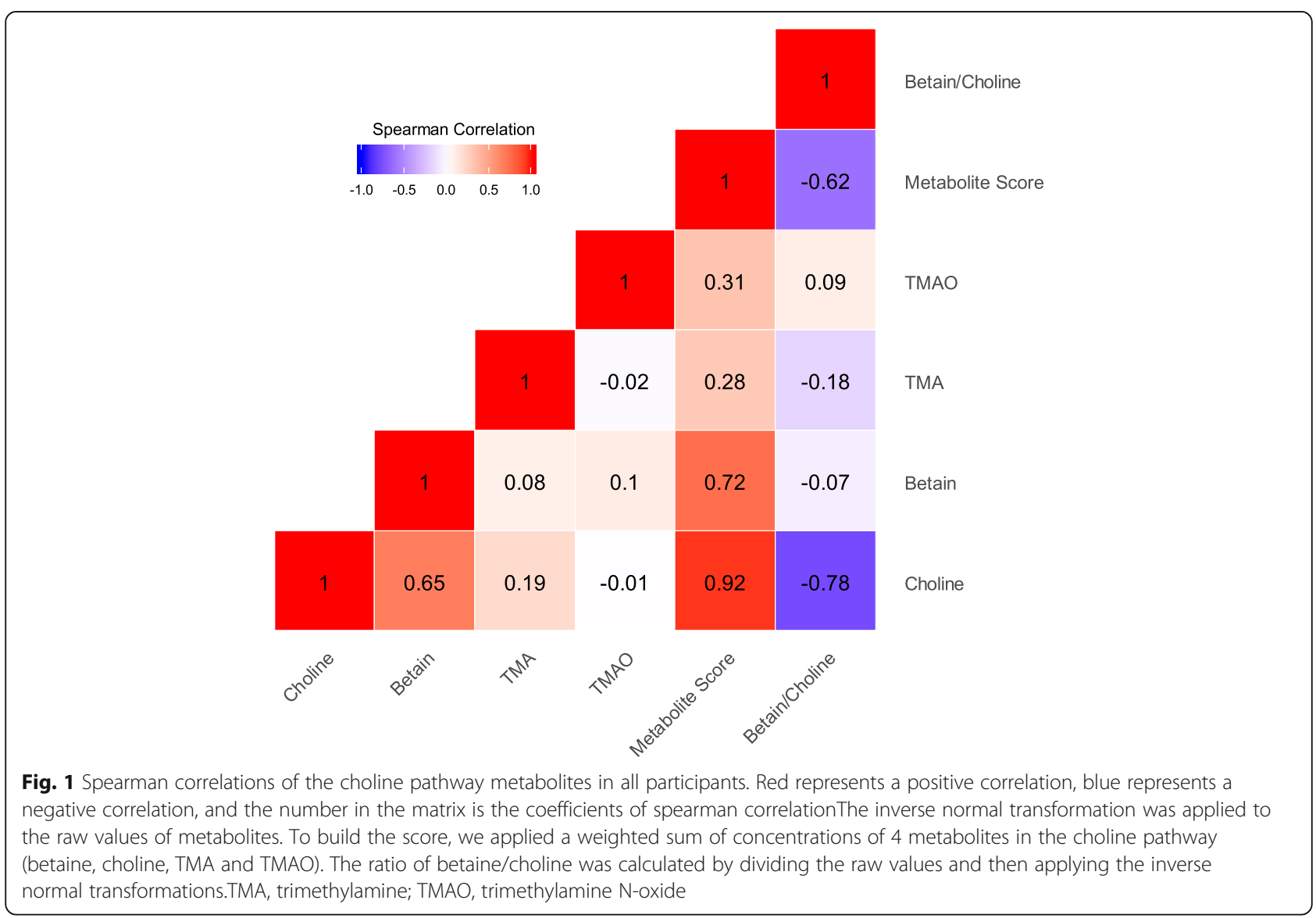


Table 2 Odds of acute coronary syndrome by circulating concentration of choline pathway metabolites

\begin{tabular}{|c|c|c|c|c|c|c|}
\hline \multirow[t]{2}{*}{ Variable } & \multicolumn{4}{|c|}{ Odds Ratio ( $95 \%$ Cl) for Quartiles of Metabolites Concentration } & \multirow{2}{*}{$\begin{array}{l}P \text { for } \\
\text { trend }\end{array}$} & \multirow{2}{*}{$\begin{array}{l}\text { Odds Ratio } \\
(95 \% \text { CI) per } \\
\text { 1-SD } \\
\text { increment }\end{array}$} \\
\hline & 1 & 2 & 3 & 4 & & \\
\hline
\end{tabular}

Betaine

No. of controls

No. of cases

Model $1^{\text {a }}$

Model $2^{b}$

Model $3^{c}$

\section{Choline}

No. of controls

No. of cases

Model $1^{\text {a }}$

Model $2^{b}$

Model $3^{c}$

TMA

$\begin{array}{lll}\text { No. of controls } & 62 & 62 \\ \text { No. of cases } & 70 & 49 \\ \text { Model } 1^{\mathrm{a}} & \text { Ref. } & 1.41(0.85-2.34) \\ \text { Model } 2^{\mathrm{b}} & \text { Ref. } & 1.53(0.92-2.57) \\ \text { Model } 3^{\mathrm{c}} & \text { Ref. } & 1.71(1.01-2.91)\end{array}$

No of controls

62

No. of cases

Model $1^{\text {a }}$

Model $2^{b}$

Model $3^{c}$

Metabolite Score

No. of controls
No. of cases
Model $1^{\text {a }}$
Model $2^{b}$
Model $3^{c}$

\section{Betaine/choline ratio}

\begin{tabular}{|c|c|c|c|}
\hline No. of controls & 62 & 62 & 61 \\
\hline No. of cases & 160 & 39 & 24 \\
\hline Model $1^{\text {a }}$ & Ref. & $0.23(0.14-0.39)$ & $0.16(0.09-0.29)$ \\
\hline Model $2^{b}$ & Ref. & $0.24(0.14-0.39)$ & $0.17(0.09-0.30)$ \\
\hline Model $3^{c}$ & Ref. & $0.24(0.14-0.41)$ & $0.17(0.09-0.30)$ \\
\hline
\end{tabular}

The inverse normal transformation was applied to the raw values of metabolites. To build the score, we applied a weighted sum of concentrations of 4 metabolites in the choline pathway (betaine, choline, TMA, and TMAO). The ratio of betaine/choline was calculated by dividing the raw values and then applying the inverse normal transformations

TMA trimethylamine, TMAO trimethylamine $\mathrm{N}$-oxide. Ref., i.e., reference group, and we used participants assigned to the first quartile of the concentrations of each metabolite, the metabolite score and betaine to choline ratio, as the reference group in each model

a in model 1, odds ratio was adjusted for age, sex, smoking index and body mass index;

${ }^{b}$ in model 2, odds ratio was adjusted for all factors in model 1, plus history of the disease (i.e., hypertension, diabetes mellitus and hyperlipidemia);

c in model 3 , odds ratio was adjusted for all factors in model 2, plus kidney function measured by eGFR
62

97

$1.43(0.87-2.34)$

0.07

$1.11(0.93-1.33)$

$1.43(0.87-2.36)$

1.12(0.93-1.35)

$1.41(0.85-2.34)$

0.08

1.12(0.93-1.34)
164

4.36 (2.61-7.38)

$<0.001$

$<0.001$

$<0.001$

62

76

$0.67(0.39-1.15)$

0.02

$1.11(0.92-1.34)$

$0.76(0.44-1.32)$

0.07

1.05(0.87-1.27)

$1.10(0.90-1.33)$

1.85(1.52-2.28)

$1.79(1.47-2.21)$

1.77(1.44-2.18) $\ldots$

0.87(0.72-1.05)

0.84(0.69-1.02)

$0.80(0.65-0.97)$ 
CI\%), 3.18 (1.85-5.54), $p<0.001$ for trend]. Per 1-SD increment in choline metabolite score was associated with higher odds of ACS [OR (95 CI\%), 1.80(1.37-2.40)], and that in betaine-to-choline ratio was inversely associated with the odds of ACS [OR (95 CI\%), 0.49 (0.39-0.60)]. Neither TMA nor TMAO was associated with ACS status in our population.

\section{Relations between Gensini score and circulating metabolites in ACS cases}

The associations of circulating concentrations of choline pathway metabolites and metabolite score with Gensini score in ACS cases were shown in Table 3.Based on our results, no significant association was observed between circulating metabolites and Gensini score among ACS cases.

\section{Discussion}

In this study of Chinese participants, we observed that higher levels of single metabolite choline, as well as a composite metabolite score representing the comprehensive effect of circulating metabolites in choline pathway, were associated with higher odds of ACS. The betaine-to-choline ratio was inversely associated with the odds of ACS. To our knowledge, this was the first study to evaluate the potential association between circulating microbial metabolites in choline pathway and

Table 3 Relations between Gensini score and circulating metabolites in acute coronary syndrome patients

\begin{tabular}{|c|c|c|c|c|c|c|}
\hline \multirow[t]{2}{*}{ Variable } & \multicolumn{4}{|c|}{$\begin{array}{l}\text { Adjusted means (standard errors) of Gesini score across quartiles of } \\
\text { metabolites levesl }\end{array}$} & \multirow[t]{2}{*}{$P$ for trend } & \multirow[t]{2}{*}{$\begin{array}{l}\text { Regression coefficient } \\
(95 \% \mathrm{Cl}) \text { per } 1-\mathrm{SD} \\
\text { increment }\end{array}$} \\
\hline & 1 & 2 & 3 & 4 & & \\
\hline \multicolumn{7}{|l|}{ Betaine } \\
\hline Model $1^{a}$ & $75.7(7.68)$ & $75.0(8.33)$ & $73.7(7.75)$ & $71.1(6.37)$ & 0.57 & $-0.18(-6.12 \sim 5.75)$ \\
\hline Model $2^{b}$ & 71.8 (13.7) & $72.0(13.5)$ & $68.9(14.0)$ & $68.4(12.9)$ & 0.65 & $0.11(-5.82 \sim 6.04)$ \\
\hline Model $3^{c}$ & $71.8(13.7)$ & $72.0(13.6)$ & $68.9(14.0)$ & $68.4(12.9)$ & 0.65 & $0.11(-5.84 \sim 6.06)$ \\
\hline \multicolumn{7}{|l|}{ Choline } \\
\hline Model $1^{a}$ & $71.6(9.44)$ & $73.1(10.34)$ & $62.4(10.42)$ & 75.7 (5.29) & 0.50 & $4.32(-2.03 \sim 10.66)$ \\
\hline Model $2^{b}$ & $69.8(14.6)$ & $72.0(14.4)$ & $54.8(15.7)$ & $71.5(12.5)$ & 0.68 & $3.77(-2.63 \sim 10.16)$ \\
\hline Model $3^{c}$ & $69.8(14.7)$ & $72.0(14.4)$ & $54.8(15.7)$ & 71.5 (12.6) & 0.68 & $3.77(-2.64 \sim 10.19)$ \\
\hline \multicolumn{7}{|l|}{ TMA } \\
\hline Model $1^{a}$ & $77.0(7.44)$ & $77.8(8.20)$ & $72.7(7.57)$ & $69.3(6.63)$ & 0.30 & $-3.78(-10.08 \sim 2.52)$ \\
\hline Model $2^{b}$ & $78.2(13.7)$ & $74.8(13.8)$ & 72.9 (13.6) & $63.6(12.8)$ & 0.11 & $-5.40(-11.85 \sim 1.06)$ \\
\hline Model $3^{c}$ & $78.3(13.7)$ & $74.7(13.8)$ & $72.8(13.7)$ & $63.5(12.9)$ & 0.11 & $-5.56(-12.13 \sim 1.00)$ \\
\hline \multicolumn{7}{|l|}{ TMAO } \\
\hline Model $1^{a}$ & $73.0(6.47)$ & $79.0(7.59)$ & $66.8(8.68)$ & $73.4(7.36)$ & 0.80 & $-1.28(-7.84 \sim 5.27)$ \\
\hline Model $2^{b}$ & $67.3(13.4)$ & 76.0 (13.3) & $62.5(14.5)$ & $68.9(14.5)$ & 0.92 & $-1.17(-7.84 \sim 5.51)$ \\
\hline Model $3^{c}$ & $67.5(13.5)$ & $76.2(13.4)$ & $62.6(14.6)$ & $68.8(13.0)$ & 0.92 & $-1.29(-8.33 \sim 5.75)$ \\
\hline \multicolumn{7}{|c|}{ Metabolite Score } \\
\hline Model $1^{\text {a }}$ & $76.7(9.82)$ & $63.0(9.01)$ & $73.4(7.46)$ & $76.2(5.87)$ & 0.55 & $3.14(-7.23 \sim 13.51)$ \\
\hline Model $2^{b}$ & $75.4(14.8)$ & $61.5(14.2)$ & $68.4(13.4)$ & $73.5(12.7)$ & 0.64 & $2.46(-7.93 \sim 12.85)$ \\
\hline Model $3^{c}$ & 75.3 (14.8) & $61.3(14.3)$ & $68.4(13.5)$ & 73.5 (12.7) & 0.64 & $2.49(-7.99 \sim 12.96)$ \\
\hline \multicolumn{7}{|c|}{ Ratio of betaine/choline } \\
\hline Model $1^{a}$ & $76.2(5.33)$ & $71.1(8.55)$ & 79.7 (11.19) & $58.4(9.83)$ & 0.15 & $-4.9(-11.21 \sim 1.41)$ \\
\hline Model $2^{b}$ & $73.7(13.0)$ & $68.7(13.3)$ & $78.9(15.6)$ & $58.4(15.0)$ & 0.26 & $-4.15(-10.55 \sim 2.25)$ \\
\hline Model $3^{c}$ & $73.7(13.0)$ & $68.5(13.4)$ & $78.8(15.7)$ & $58.4(15.0)$ & 0.26 & $-4.15(-10.56 \sim 2.26)$ \\
\hline \multicolumn{7}{|c|}{$\begin{array}{l}\text { Inverse normal transformation was applied to raw values of metabolites. To build the score, we applied a weighted sum of concen } \\
\text { choline pathway (betaine, choline, TMA and TMAO). The ratio of betaine/choline was calculated by dividing the raw values and th } \\
\text { transformations } \\
\text { TMA, trimethylamine; TMAO, trimethylamine N-oxide } \\
\text { a Model } 1 \text { was adjusted for age, sex, smoking index and body mass index } \\
\text { b Model } 2 \text { was adjusted for all factors in model 1, plus history of disease (i.e., hypertension, diabetes mellitus and hyperlipidemia) } \\
\text { c Model } 3 \text { was adjusted for all factors in model 2, plus kidney function measured by eGFR }\end{array}$} \\
\hline
\end{tabular}


ACS in Chinese population, and may provide novel perspectives on the choline metabolites and CVD.

It has long been known that habitual dietary pattern is a significant contributor to the CVD risk [17]. Gut microbial metabolism of dietary nutrients may result in the production of proatherogenic circulating factors that act through a meta-organismal endocrine axis to impact CVD risk [18]. For example, circulating metabolites in the choline pathway are derivatives from dietary nutrients, and the gut microbiome is actively involved in its metabolism pathway [19]. Our findings suggest that circulating metabolites in the choline pathway may play a role in the ACS pathophysiology in Chinese, reflecting a link between dietary metabolism, gut microbiome and ACS development. Several mechanisms, such as pro-atherogenic and prothrombotic effects can at least partially explain these associations between metabolites and ACS [2, 7].

There is accumulating evidence of the associations between TMAO and cardiovascular events, and a metaanalysis of 19 cohorts suggested a positive association of TMAO and its precursors with the risk of major adverse cardiovascular endpoints [8]. However, it should be noted that most cohorts were conducted in western populations, while the western diet patterns contain abundant nutrient precursors for TMAO [7]. Choline-rich foods (such as red meat, egg yolks, and milk) are converted by the gut microbiota into TMA, which is further oxidized into TMAO in the liver by the hepatic flavincontaining monooxygenase family of enzymes [20]. Chronic consumption of diets rich in red meat increased both plasma and urine TMAO levels [21]. In this study of a Chinese population, the median of TMAO levels was $1.45 \mu \mathrm{mol} / \mathrm{L}$ (interquartile range: $0.94-2.32$ ), which was lower than that in western populations [7, 15, 22], and similar to that in another study conducted in Chinese populations [23].

Previous studies in western populations have shown that TMAO levels were related to atherosclerosis and CVD, and reducing TMAO production may be a potential therapeutic target for CVD [2, 3, 24]. However, the association between TMAO and adverse outcomes was distinctive in different races [25]. Moreover, a recent bi-directional Mendelian randomization analysis suggested that TMAO levels were elevated in patients of type 2 diabetes and chronic kidney disease, and that confounding or reverse causality should also be considered when explaining the previously reported associations [26]. In the current study, we did not found a significant association between circulating TMAO concentration and ACS. Factors such as the differences in dietary choline intake, intestinal microbiota activity and flavin monooxygenase activity, and the divergent concentrations of TMAO in different populations as mentioned above, may explain the null association between TMAO and ACS through complex mechanisms in our Chinese population.
As the metabolic precursors of TMAO, choline (the precursor of betaine) and betaine have also been linked with the risk of CVD in several studies [15, 27-30]. The major fate of dietary choline is conversion to phosphatidylcholine, which is essential in the secretion of verylow-density lipoproteins from the liver [31]. Betaine is a determinant for homocysteine concentration, which may increase abnormal platelet activity and blood coagulation, leading to thrombotic disorders such as ACS [32]. In an ACS cohort study, plasma betaine was found negatively correlated with triglyceride and non-high-density lipoprotein cholesterol and low plasma betaine concentrations contributed to metabolic syndrome and known prognostic markers of vascular diseases [29, 30]. However, a systematic review and meta-analysis of six prospective studies from three countries (USA, Japan, and The Netherlands) did not suggest the associations of choline or betaine with incident CVD or CVD mortality [27]. In our study, we did not find a statistically significant association between blood betaine levels and ACS, although the betaine/choline ratio was inversely associated with the odds of ACS. Our results in a Chinese population are consistent with that in a case-control study conducted within the PREDIMED (Prevention With Mediterranean Diet) trial in the Spanish populations [15]. Because the betaine/choline ratio combines the predictive power of the choline (precursor) and betaine (product) pathway, it may further state the importance of the choline pathway in the ACS pathogenesis. Platelet activity, blood coagulation and lipid profile may be the underlying mechanisms [32].

Several limitations of this analysis are noteworthy. First, the causality of the observed relationship cannot be inferred because of the case-control study design. In particular, residual confounding such as medication use cannot be excluded. Second, the participants were patients who visited Zhongshan hospital and were mostly unhealthy in general. Thus, our results might not be generalizable to the general Chinese population. Third, we did not collect dietary information or fecal samples for each participant, and therefore could not explore the complete association among dietary intake, gut microbiome and circulating metabolites.

\section{Conclusion}

The present study suggested that circulating choline levels were higher in patients with ACS among Chinese participants. A higher composite metabolite score of metabolites from the choline pathway, which were related to the gut microbiome, was associated with increased odds of ACS in Chinese participants. The choline pathway may be related to the pathophysiology of ACS, and further mechanistic studies are warranted. 


\section{Abbreviations}

ACS: Acute coronary syndrome; CVD: Cardiovascular disease: TMAO: Trimethylamine N-oxide; TMA: Trimethylamine; eGFR: Estimated glomerular filtration rate; LC-MS: Liquid chromatography tandem mass spectrometry; OR: Odds ratio; Cl: Confidence intervalBMIBody mass index

\section{Acknowledgments}

We would like to thank all the participants and the research staff at Zhongshan hospital.

\section{Authors' contributions}

YD and QT contributed equally to this work. YZ and GJ conceived and designed the study; YD, SS, LX, DR, SC and GJ recruited the participants and collected the data; XD and HZ performed all the metabolic analyses; QT, ZS and ZM conducted the statistical analyses of the data; QT, YD and JS wrote the first draft of manuscript, and all authors reviewed and revised the manuscript. All authors read and approved the final manuscript.

\section{Funding}

This project was supported by the National Natural Science Foundation of China (Grant No. 31741072 and 81973032), Shanghai Municipal Science and Technology Major Project (Grant No. 2017SHZDZX01), and the National Key Research and Development Program of China (Grant No. 2016YFC1301200). YZ was supported by the Program for Professor of Special Appointment (Eastern Scholar) at Shanghai Institutions of Higher Learning. YD was supported by the National Natural Science Foundation of China (Grant No. 81300095), Animal Model Project of Shanghai Scientific committee (Grant No. 19140900901) and Youth Backbone Foundation of Zhongshan Hospital. The funding agencies had no role in the design and conduct of the study, in the collection, management, analysis, and interpretation of the data, or in the preparation, review, or approval of the manuscript, or decision to submit for publication.

\section{Availability of data and materials}

The datasets used and/or analysed during the current study are available from the corresponding authors on reasonable request.

\section{Ethics approval and consent to participate}

All participants gave informed consents after they received information about the study and the voluntary nature of their participation and before any measurements were performed. The Ethical Committee of Zhongshan Hospital Fudan University approved this study, and all the participants provided written informed consent.

\section{Consent for publication}

Not Applicable.

\section{Competing interests}

The authors declare no conflicts of interest.

\section{Author details}

'Department of Cardiology, Shanghai Institute of Cardiovascular Disease, ZhongShan Hospital, Fudan University, 1609 Xietu Road, Shanghai 200032, China. ${ }^{2}$ Department of Anthropology and Human Genetics, School of Life Sciences, Fudan University, Shanghai, China. ${ }^{3}$ School of Kinesiology, Shanghai University of Sport, Shanghai, China. ${ }^{4}$ Human Phenome Institute, Fudan University, 2005 Songhu Road, Shanghai 200438, China. Institute of translational medicine, Shanghai University, Shanghai, China.

\section{Received: 21 February 2020 Accepted: 7 May 2020}

\section{Published online: 24 May 2020}

\section{References}

1. GBD 2013 Mortality and Causes of Death Collaborators. Global, regional, and national age-sex specific all-cause and cause-specific mortality for 240 causes of death, 1990-2013: a systematic analysis for the Global Burden of Disease Study 2013. Lancet. 2015;385(9963):117-71.

2. Wang Z, Klipfell E, Bennett BJ, Koeth R, Levison BS, DuGar B, et al. Gut flora metabolism of phosphatidylcholine promotes cardiovascular disease. NATU RE. 2011;472(7341):57-63.
3. Tang WHW, Wang Z, Levison BS, Koeth RA, Britt EB, Fu X, et al. Intestinal microbial metabolism of Phosphatidylcholine and cardiovascular risk. N Engl J Med. 2013;368(17):1575-84.

4. Koeth RA, Wang Z, Levison BS, Buffa JA, Org E, Sheehy BT, et al. Intestinal microbiota metabolism of $\mathrm{L}$-carnitine, a nutrient in red meat, promotes atherosclerosis. Nat Med. 2013;19(5):576-85.

5. lerardi E, Sorrentino C, Principi M, Giorgio F, Losurdo G, Di Leo A. Intestinal microbial metabolism of phosphatidylcholine: a novel insight in the cardiovascular risk scenario. Hepatobiliary Surg Nutr. 2015;4(4):289-92.

6. Tang WH, Wang Z, Shrestha K, Borowski AG, Wu Y, Troughton RW, et al. Intestinal microbiota-dependent phosphatidylcholine metabolites, diastolic dysfunction, and adverse clinical outcomes in chronic systolic heart failure. J Card Fail. 2015;21(2):91-6.

7. Li XS, Obeid S, Klingenberg R, Gencer B, Fo M, Räber L, et al. Gut microbiota-dependent trimethylamine $\mathrm{N}$-oxide in acute coronary syndromes: a prognostic marker for incident cardiovascular events beyond traditional risk factors. Eur Heart J. 2017:38(11):814-24.

8. Heianza Y, Ma W, Manson JE, Rexrode KM, Qi L. JJotAHAC, Disease CGut Microbiota Metabolites and Risk of Major Adverse Cardiovascular Disease Events and Death: A Systematic Review and Meta-Analysis of Prospective Studies. J Am Heart Assoc. 2017:6(7):e004947.

9. Schiattarella GG, Sannino A, Toscano E, Giugliano G, Gargiulo G, Franzone A, et al. Gut microbe-generated metabolite trimethylamine-N-oxide as cardiovascular risk biomarker: a systematic review and dose-response metaanalysis. Eur Heart J. 2017;38(39):2948-56.

10. Roffi M, Patrono C, Collet J-P, Mueller C, Valgimigli M, Andreotti F, et al. 2015 ESC guidelines for the management of acute coronary syndromes in patients presenting without persistent ST-segment elevation: task force for the Management of Acute Coronary Syndromes in patients presenting without persistent ST-segment elevation of the European Society of Cardiology (ESC). Eur Heart J. 2016;37(3):267-315.

11. Ibanez B, James S, Agewall S, Antunes MJ, Bucciarelli-Ducci C, Bueno H, et al. 2017 ESC guidelines for the management of acute myocardial infarction in patients presenting with ST-segment elevation: the task force for the management of acute myocardial infarction in patients presenting with ST-segment elevation of the European Society of Cardiology (ESC). Eur Heart J. 2017;39(2):119-77.

12. Gensini GG. A more meaningful scoring system for determining the severity of coronary heart disease. Am J Cardiol. 1983;51(3):606.

13. Patel RS, Su S, Neeland IJ, Ahuja A, Veledar E, Zhao J, et al. The chromosome 9p21 risk locus is associated with angiographic severity and progression of coronary artery disease. Eur Heart J. 2010;31(24):3017-23.

14. Beasley TM, Erickson S, Allison DB. Rank-based inverse normal transformations are increasingly used, but are they merited? Behav Genet. 2009:39(5):580-95.

15. Guasch-Ferré M, Hu FB, Ruiz-Canela M, Bulló M, Toledo E, Wang DD, et al. Plasma metabolites from choline pathway and risk of cardiovascular disease in the PREDIMED (prevention with Mediterranean diet) study. J Am Heart Assoc. 2017;6(11):e006524.

16. Yan J, Winter LB, Burns-Whitmore B, Vermeylen F, Caudill MA. Plasma choline metabolites associate with metabolic stress among young overweight men in a genotype-specific manner. Nutrition Diabetes. 2012;2(10):e49.

17. Yu E, Rimm E, Qi L, Rexrode K, Albert CM, Sun Q, et al. Diet, lifestyle, biomarkers, genetic factors, and risk of cardiovascular disease in the Nurses' health studies. Am J Public Health. 2016;106(9):1616-23.

18. Brown JM, Hazen SL. The gut microbial endocrine organ: bacterially derived signals driving cardiometabolic diseases. Annu Rev Med. 2015;66:343-59.

19. Tang WHW, Hazen SL. The contributory role of gut microbiota in cardiovascular disease. J Clin Invest. 2014;124(10):4204-11.

20. Wang Z, Roberts AB, Buffa JA, Levison BS, Zhu W, Org E, et al. Non-lethal inhibition of gut microbial Trimethylamine production for the treatment of atherosclerosis. Cell. 2015;163(7):1585-95.

21. Wang Z, Bergeron N, Levison BS, Li XS, Chiu S, Jia X, et al. Impact of chronic dietary red meat, white meat, or non-meat protein on trimethylamine $\mathrm{N}$ oxide metabolism and renal excretion in healthy men and women. Eur Heart J. 2019:40(7):583-94.

22. Senthong $\mathrm{V}$, Wang Z, Fan $\mathrm{Y}, \mathrm{Wu} \mathrm{Y}, \mathrm{WHW}$ T. JJotAHATrimethylamine $\mathrm{N} \mathrm{m}^{2}$ xide and Mortality Risk in Patients With Peripheral Artery Disease. J Am Heart Assoc. 2016;5(10):e004237.

23. Sheng Z, Tan Y, Liu C, Zhou P, Li J, Zhou J, et al. Relation of circulating Trimethylamine $\mathrm{N}$-oxide with coronary atherosclerotic burden in patients 
with ST-segment elevation myocardial infarction. Am J Cardiol. 2019:123(6):894-8.

24. Landfald B, Valeur J, Berstad A, Raa J. JMEiH, DiseaseMicrobial trimethylamine-N-oxide as a disease marker: something fishy? Microb Ecol Health Dis. 2017;28(1):1327309.

25. Shafi T, Powe NR, Meyer TW, Hwang S, Hai X, Melamed ML, et al. Trimethylamine-oxide and cardiovascular events in hemodialysis patients. J Am Soc Nephrol. 2017;28(1):321.

26. Jia J, Dou P, Gao M, Kong X, Li C, Liu Z, et al. Assessment of causal direction between gut microbiota-dependent metabolites and Cardiometabolic health: a bidirectional Mendelian randomization analysis. Diabetes. 2019; 68(9):1747.

27. Katie M, Jonathan S. Dietary Choline and Betaine and Risk of CVD: A Systematic Review and Meta-Analysis of Prospective Studies. Nutrients. 2017;9(7):711.

28. Millard HR, Musani SK, Dibaba DT, Talegawkar SA, Taylor HA, Tucker KL, et al. Dietary choline and betaine; associations with subclinical markers of cardiovascular disease risk and incidence of CVD, coronary heart disease and stroke: the Jackson heart study. Eur J Nutr. 2018;57(1):51-60.

29. Lever M, George PM, Elmslie JL, Atkinson W, Slow S, Molyneux SL, et al. Betaine and secondary events in an acute coronary syndrome cohort. PLoS One. 2012;7(5):e37883.

30. Lever M, George PM, Atkinson W, Molyneux SL, Elmslie IL, Slow S, et al. Plasma lipids and Betaine are related in an acute coronary syndrome cohort. PLoS One. 2011;6(7):e21666.

31. Ueland PM. Choline and betaine in health and disease. J Inherit Metab Dis. 2011;34(1):3-15.

32. Nagata C, Wada K, Tamura T, Konishi K, Kawachi T, Tsuji M, et al. Choline and Betaine intakes are not associated with cardiovascular disease mortality risk in Japanese men and women. J Nutr. 2015;145(8):1787-92.

\section{Publisher's Note}

Springer Nature remains neutral with regard to jurisdictional claims in published maps and institutional affiliations.

Ready to submit your research? Choose BMC and benefit from:

- fast, convenient online submission

- thorough peer review by experienced researchers in your field

- rapid publication on acceptance

- support for research data, including large and complex data types

- gold Open Access which fosters wider collaboration and increased citations

- maximum visibility for your research: over $100 \mathrm{M}$ website views per year

At $\mathrm{BMC}$, research is always in progress.

Learn more biomedcentral.com/submissions 